\title{
Design and implementation of vehicle intelligent terminal based on vehicle networking and intelligent technology
}

\author{
Li Hong $^{1}$, Shi Guoyou ${ }^{2}$ \\ ${ }^{1}$ Department of Automotive Engineering,Beijing Information Technology College,Beijing \\ 100070,China; \\ ${ }^{2}$ Department of Technical Protection,Beijing Archives Bureau, Beijing 100070,China
}

Key words: Vehicle networking; intelligent technology; intelligent terminal; CAN bus; bus

\begin{abstract}
Vehicle terminal is the main information source of vehicle networking, and also the key technology. Combining GPS, GPRS, CAN bus, sensors and embedded technology advantages, and based on the theory of public transportation intelligent terminal system, the design method of car networking and intelligent terminal based on intelligent technology has been put forward in this paper, it has realized the whole scheme design of bus borne intelligent terminal, automatic station reporting and positioning function, map navigation function, voice navigation function, intelligent navigation function and so on. The realization of the terminal can improve the optimization and improvement of the vehicle intelligent terminal.
\end{abstract}

\section{Introduction}

With the development of GPS technology, artificial intelligence, computer control technology and human-computer collaborative technology by leaps and bounds, car navigation, voice broadcast system has been successfully applied in the high-end car in the future will also be applied to the low-end car. Intelligent terminal vehicle collection, networking, networking, intelligent technology, GPS, GSM, GPRS, sensor technology, Linux systems, ARM and other technologies in one, to improve the operation and management of public transport systems have greater help. This paper takes Exynos 4412 processor as the core, combined with the car networking, networking technology, sensor technology and embedded control, the automatic positioning, bus map updating and voice broadcast function has been realized in the research and design of the vehicle terminal.

\section{Integrated scheme design of bus intelligent terminal}

Vehicle intelligent terminal function description. (1) automatic reporting station: the system is added to the GPS automatic positioning system, and it is automatically reported according to the site coordinate information.

(2) positioning function: GPS can accurately determine its location by receiving satellite signals, and the position error is less than 10 meters. Use GPS to display the current position of the vehicle in real time on the map;

(3) map navigation function according to the starting point and end point set, automatically according to the real-time traffic planning for the driver route; if the driver accidentally deviates from the line, according to the current route planning an optimal route, or for the terminal customers to its current position as a starting point to the session, the final destination for the end point, again planning the best route to the new.

(4) voice navigation function: after entering the target location, the vehicle as long as the front of the intersection or turn, the car GPS voice system prompts the user to turn and other voice prompts; 
(5) entertainment function: play U disk, SD card in the MP3, MP4 files;

(6) intelligent navigation function: when the GPS signal is lost in a short time, the vehicle can be calculated according to the acceleration sensor in the vehicle and the speed of the vehicle;

(7) increase the interest point function: you can add the latest updated route or building on the map independently.

Whole scheme design. SAMSUNG Exynos 4412 processor has been used for the core processor in this control system, which uses high performance integrated peripheral design, the main frequency is $1.4 \mathrm{G}$, the processing capacity is super, and it is also equipped with rich on-chip resources. According to the special requirement of the intelligent terminal of the bus and the characteristics of the Exynos 4412 processor, the scheme of the intelligent terminal system for the public transportation vehicle is designed. The main functional frame of the system is shown in figure 1.

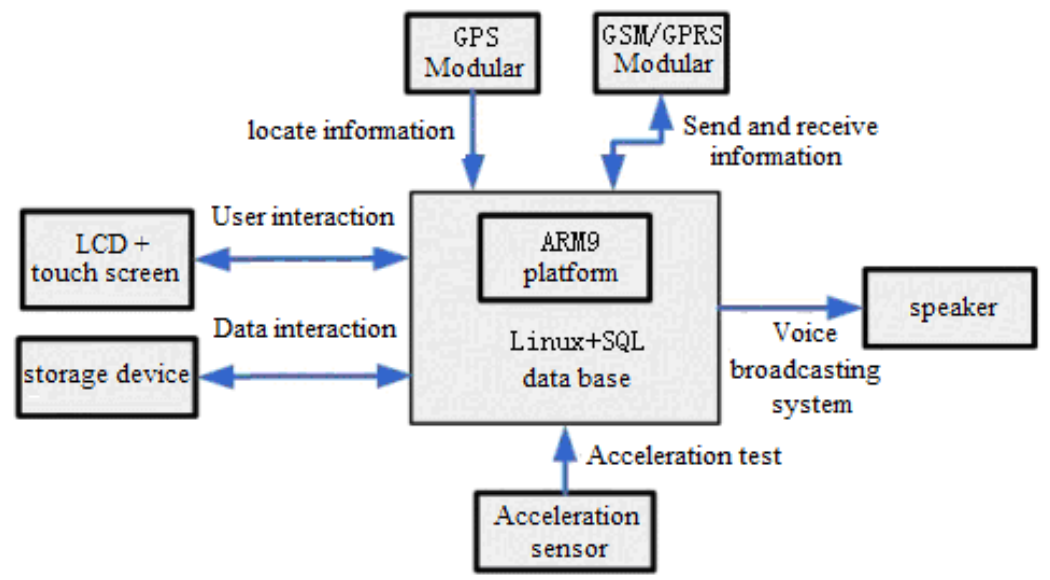

Figure 1 function diagram of intelligent terminal

As shown in Figure 1, ARM9 system is used in the Exynos 4412 processor, so the system on the ARM9 platform and the Linux platform, and the platform is designed based on the intelligent terminal in GPS positioning module, GSM/GPRS transceiver module, voice broadcast system, three axis accelerometer integrated control system.

\section{System hardware design}

(1) Design of power supply module;

The Exynos 4412 processor is adopted as the main control unit in the intelligent terminal control system, which has higher power performance requirements, so the $3.3 \mathrm{~V}$ and $1.8 \mathrm{~V}$ dual power supply is used. The power supply is introduced from an external regulated power supply to the $5 \mathrm{~V}$ voltage, and then the buck is respectively depressed by the CAT6219-180TD-GT3 and the AMS1084CM-3.3 voltage regulator module. The output voltage is $3.3 \mathrm{~V}$ and $1.8 \mathrm{~V}$. The principle diagram of the power module of the intelligent terminal control system is shown in figure 2 . 


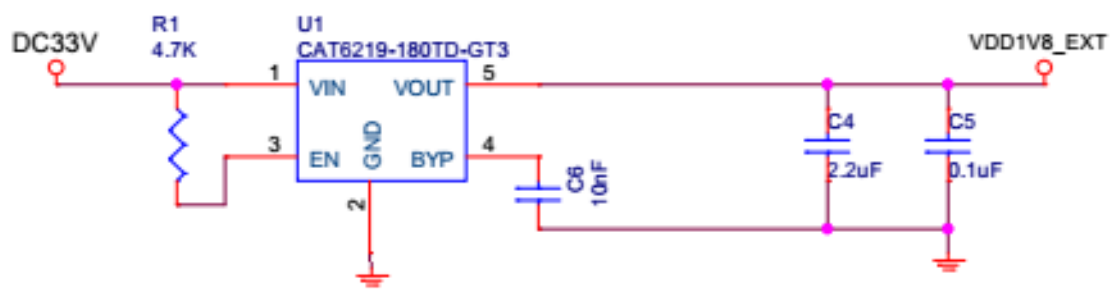

(2) CAN bus design

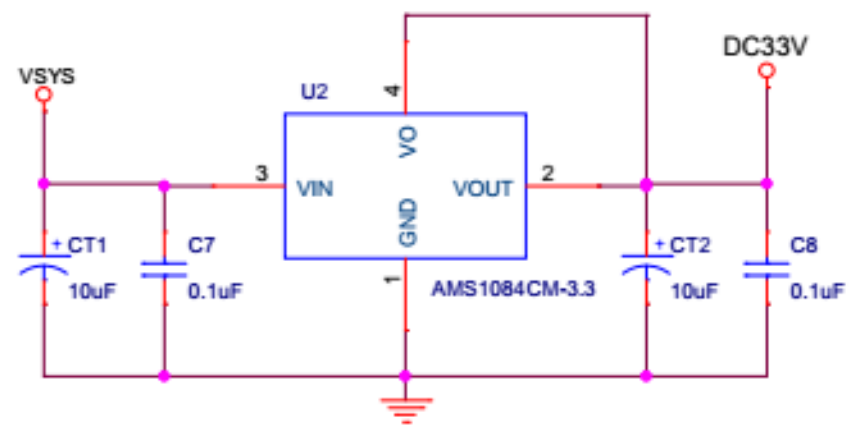

Fig. 2 Schematic diagram of power supply module

MCP2515 controller is a built-in CAN bus protocol, as long as the external bus driver chip and appropriate anti-interference circuit can easily build a CAN bus intelligent node, its high performance, high reliability and unique design and reasonable price and is widely used in industrial control, intelligent buildings, medical equipment, vehicles and sensors etc.. The schematic diagram of the CAN bus circuit is shown in figure 3.

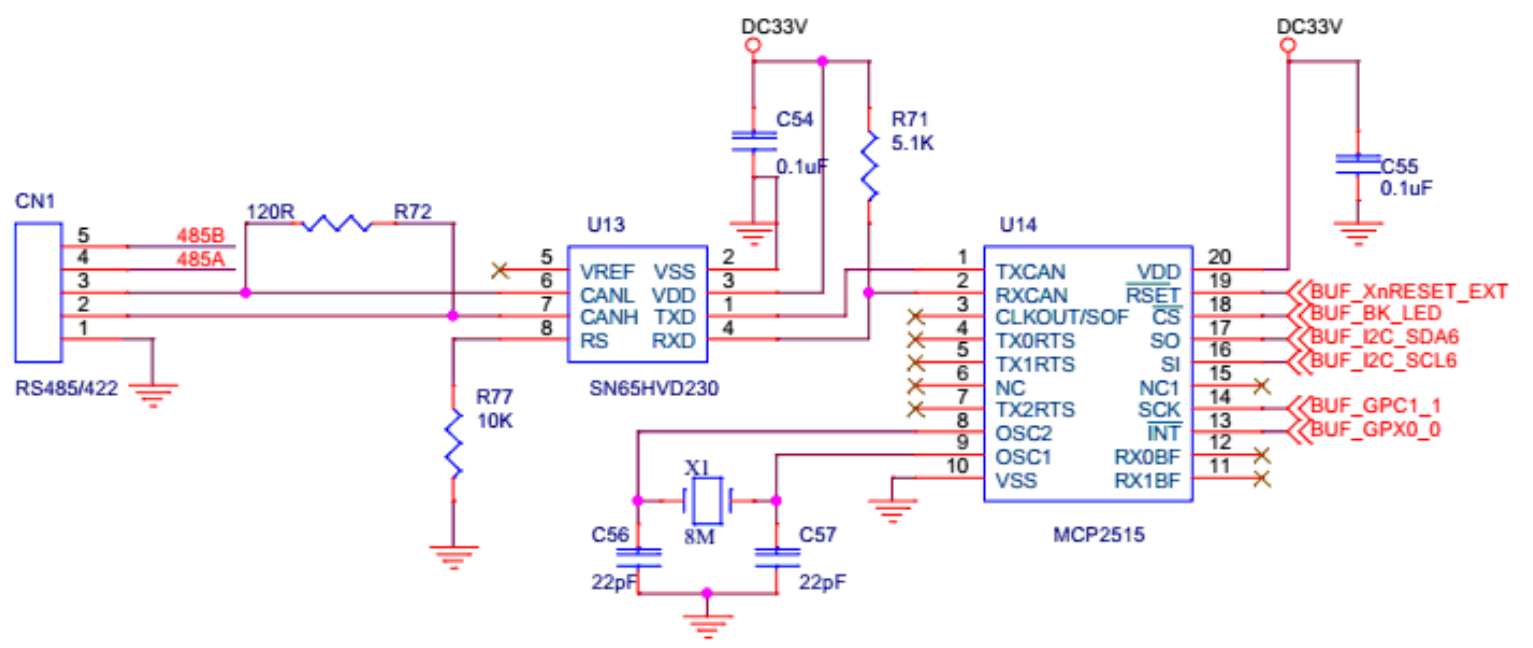

Fig. 3 Schematic diagram of CAN bus circuit

(3) Design of three axis gravity sensor circuit

The register of the three axis gravity sensor MPU6050 is the same as the register of three sensors, such as temperature, gyroscope, and external sensing data, which are composed of two registers, which are invisible to the user inside and outside of the user. The data in the internal registers in the sampling time to timely update, only in the serial communication interface is not busy, the internal value of the register is copied to the user readable registers to avoid direct access to emergency induction measurements. In the process of using, the general is raw data collected by MPU6050, after the CPU process can get the pitch angle and roll angle, the data integration in electronic compass can be heading Yaw, which can get three axis change data. The schematic diagram of the application is shown in Figure 4. 


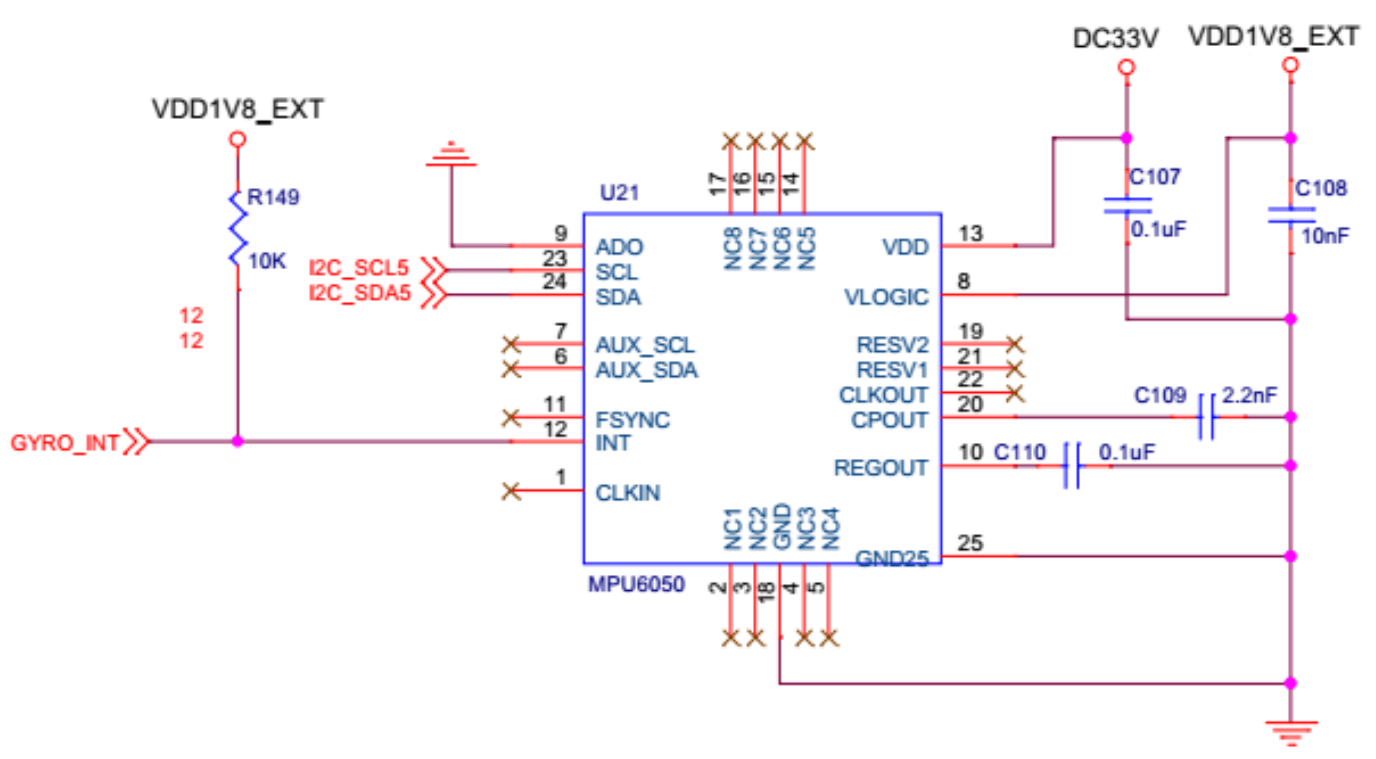

Fig.4 The application schematic diagram of MPU6050

\section{System software design}

The bus vehicle intelligent terminal system software in this paper adopts object-oriented and modular design, focus on intelligent terminal positioning system accuracy and reliability as well as the voice broadcast humanization design in the development stage. Using the Keil Uvision4 platform development environment, Keil Uvision4 provides a powerful development environment for the Exynos 4412 processor, which integrates a large number of device driver source code. In the process of software design, modularization design can be realized simply by simple calling, which provides great convenience for the development and management of Exynos 4412 embedded engineering project. The system software includes Main main program and GPS positioning module, GSM/GPRS information transceiver module, voice broadcast system, three axis gravity acceleration sensor, electronic map and other subroutines. The main program realizes the normal operation of the vehicle intelligent terminal by using the sub program function entrance. The software flowchart of the vehicle intelligent terminal control system is shown in Figure 5.

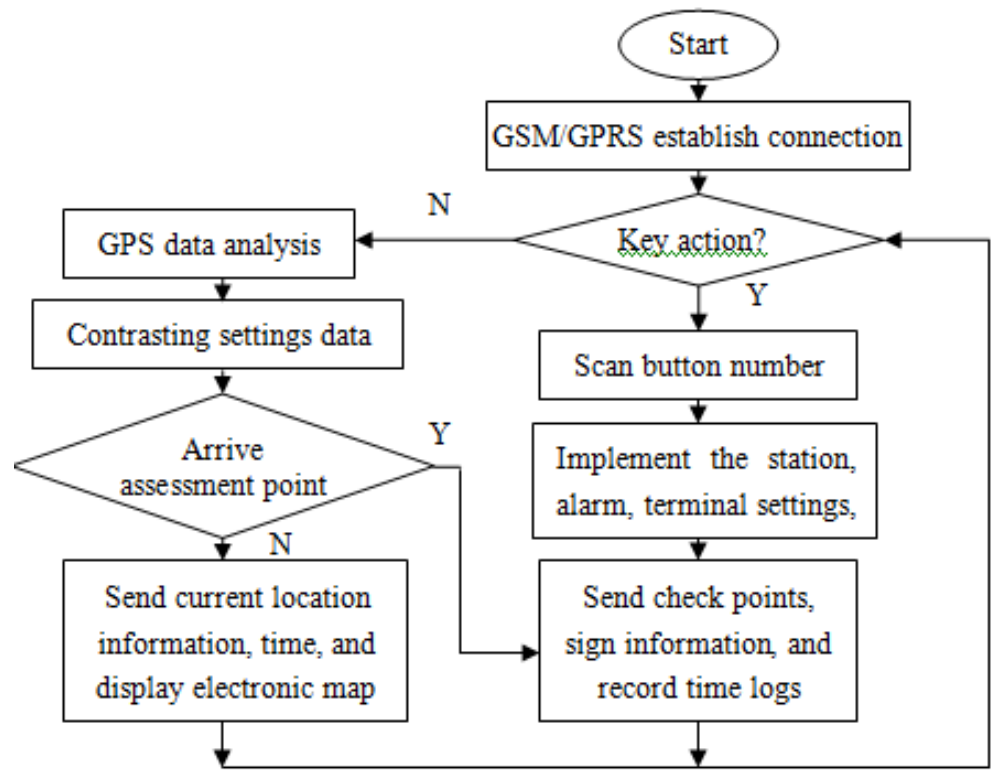

Fig. 5 Software flow chart of vehicle intelligent terminal control system 
Design of three axis gravity sensor software is a subroutine design more complex, MPU6050 respectively with three 16 bit ADC of gyroscope and accelerometer, the measurement of analog quantity into digital quantity output, in order to accurately track vehicle speed sensor, the measurement range is user controllable. The module code is as follows:

int main(void)

\{

REG_WRITE(GPX1PUD,REG_READ(GPX1PUD) \& $(\sim(0 \mathrm{x} 3<<2)))$;

REG_WRITE(GPX1CON,REG_READ(GPX1CON) \& $(0 \mathrm{xf}<<4) \mid(0 \mathrm{xf}<<4))$;

REG_WRITE(EXT_INT41_CON,REG_READ(EXT_INT41_CON) \& $(0 x 7<<4) \mid(0 x 2$ $<<4)$;

REG_WRITE(EXT_INT41_MASK,REG_READ(EXT_INT41_MASK) \& $(\sim(0 x 1<<1)))$;

REG_WRITE(ICDDCR,1);

REG_WRITE(ICDISER1_CPU0,REG_READ(ICDISER1_CPU0) \& $(\sim(0 x 1<<25)) \mid$ $(1<<25))$; $(1<<8))$;

REG_WRITE(ICDIPTR14_CPU0,REG_READ(ICDIPTR14_CPU0) \& $(\sim(0 x f f<<8))$ |

REG_WRITE(ICCICR_CPU0,1);

REG_WRITE(ICCPMR_CPU0,0xff);

REG_WRITE(GPX2CON,REG_READ(GPX2CON) \& $(\sim(0 \mathrm{xf}<<28)) \mid(0 \mathrm{x} 1<<28))$;

REG_WRITE(GPX2DAT,REG_READ(GPX2DAT) \& $(\sim(0 \mathrm{x} 1<<7)))$;

i2c5_init();

mydelay_ms(100);

uart2_init();

mydelay_ms(100);

MPU6050_Init();

mydelay_ms(100);

return 0 ;

\}

\section{Conclusion}

This paper is based on the bus vehicle intelligent terminal system theory, combined with the characteristics of bus driving and intelligent control system, the overall design scheme of the design and research of the system, an important circuit on the three axis gravity sensor circuit design, CAN design, the design of power module are introduced, the corresponding platform for. Each subsystem of the modular programming, finally realizes the monitoring and scheduling of public transport vehicles, and automatic terminal station, positioning function, map navigation function, voice navigation, intelligent navigation function, and has important significance and improvement of the intelligent terminal bus.

\section{References}

[1] $\mathrm{Hu}$ Weimin, Chen Ping, Xie Qiming, Fu Liang. Design and implementation of intelligent transportation system based on Internet of things technology [J]. Computer Era, No. 09, (2016), p.51-55. 
[2] Fan Yuxiao, Xie Jinfeng. Research on standard system of vehicle information terminal [J]. Digital Communication, No. 06, (2014), p.28-31.

[3] Huang Zhengfeng, Ren Gang, Hua Jingyi, Lu Lili. Design of bus information perception mining system based on distributed processing technology [J]. Urban Transport of China, No.05, (2014), p.60-64.

[4] Wang Lei. Telematics. Application in vehicle navigation system [D]. Dalian University of Technology, (2014).

[5] Wang Sen. Research on endoscopic tracking and positioning system based on MEMS sensor fusion technology [D]. Tianjin University, (2014).

[6] Hou Lixin. Anhui University design and implementation of [D]. intelligent bus platform based on RT-Thread, (2013).

[7] Du Cheng. Design and implementation of vehicle navigation and monitoring system based on GPS and GIS[D]. Southwest Jiao Tong University, (2005).

[8] Hu Xiong. Application of GPS in automobile [J]. Technology and enterprise, Vol.15, (2012) , p.127.

[9] Luo Le. Design and implementation of logistics vehicle GPS intelligent management terminal [J]. Logistics Technology (Equipment Edition), Vol.14, (2012) , p.48-50.

[10] Zhang Yunli. Discussion on vehicle GPS navigation system and its development prospects [J]. Science \& Technology Information, Vol.36, (2011) , p.209-210.

[11] Tang Caiyun. Design and implementation of Cartographic Symbol Library for embedded vehicle navigation system [D]. Chinese people's The PLA Information Engineering University, (2002).

[12] Huang Qingnan, Chen Wenhui, Susan. ARM7 design and implementation of intelligent public transportation vehicle system [J]. Automation \& Instrumentation, No.06, (2011) , p.16-19.

[13] Liu Zhikai. Design and implementation of intelligent vehicle terminal based on ARM [D]. Hebei University of Technology, (2011).

[14] Chen Lei. Design and implementation of 3D simulation vehicle navigation system [D]. University of Electronic Science and technology of China, (2010).

[15] Ye Fang, Tan Haiwei. Application of speed sensor based on GPS in vehicle handling stability test [J]. Journal of Guizhou University (Natural Sciences), No.02, (2010), p.119-122.

[16] Zhang Wenqian. Design and implementation of vehicle location and navigation system based on GPS/DR and GSM [D]. Harbin Engineering University, (2010).

[17] Luan Shengyuan, Design and implementation of intelligent bus terminal based on embedded VxWorks [D]. Qingdao University of Science \& Technology, (2009).

[18] Tian Yi, Liu Xiuhong. Design of CAN bus communication system based on C8051F040 [J]. Modern Electronic Technique, Vol.15, (2006) , p.29-30,33.

[19] Yang Jin. Research on Application of OPC in field bus control system [D]. Wuhan University of Technology, (2006).

[20] Yang Jinxian. Design and implementation of vehicular navigation system based on ARM-Linux platform [D]. Harbin Engineering University, (2006). 